\title{
Community-based Participatory Research (CBPR) and Refugees' Health Studies: What's Working and What's Not Working
}

\author{
Huaibo Xin \\ Department of Applied Health, Southern Illinois University Edwardsville, USA
}

Copyright $@ 2019$ by authors, all rights reserved. Authors agree that this article remains permanently open access under the terms of the Creative Commons Attribution License 4.0 International License

\begin{abstract}
The Community-based Participatory Research (CBPR) has been well defined and widely applied to different study populations in a variety of health topic areas to address the urgency of translating research into practice, reducing health disparities, improving health equity, and advocating for health policy changes. The current study aims to systematically examine how CBPR has been applied to improving underserved refugee populations' health and identify the successes and challenges of CBPR utilization among refugees. A total of 930 peer-reviewed journal articles, reports, commentaries, theses, dissertations, books, and book chapters in English, retrieved from several major databases (e.g., EBSCO, ERIC, PubMed, PsycINFO, CINAHL Plus, and Google Scholar) were initially reviewed. Fourteen peer-reviewed journal articles were finally selected and analyzed using the theme analysis. Results showed that successes of utilization CBPR in refugee health studies were achieved in areas of shared learning, trust, recruitment, methodological rigor, advocacy, sociocultural determinants, dissemination/ sustainability, ethics, cultural competency, and stigma. However, the CBPR practice was also challenged by issues emerging from institutional culture and structure, clinical procedures, non-probability sampling, self-reflection, and repetition. Current literature suggests that overcoming these challenges requires both institutional restructure and policy changes, and changes in the focus of both internal and external funding mechanisms.
\end{abstract}

Keywords Community-based Participatory Research, Photovoice, Refugees, Health

\section{Introduction}

The current literature has commonly defined Community-based Participatory Research (CBPR) as "a collaborative research approach that is designed to ensure and establish structures for participation by communities affected by the issue being studied, representatives of organizations, and researchers in all aspects of the research process to improve health and well-being through taking action, including social change [1]" as well as to reduce health disparities [2-4]. It further involves "co-learning and reciprocal transfer of expertise shared decision-making power, and mutual ownership of the processes and products of the research enterprise [1].” The keys to successfully practicing CBPR are to develop collaborative and equal relationships between researchers and community partners "at various levels within the community, valuing diverse cultural perspectives, place equal emphasis and importance on community knowledge and academic perspectives, and allow for flexibility in research methods [5]." The benefits of applying CBPR to research include but are not limited to "(a) ensuring that the research topic comes from, or reflects, a major concern of the local community; (b) enhancing the relevance and application of the research data by all partners involved; (c) bringing together partners with different skills, knowledge, and expertise to address complex problems; (d) enhancing the quality, validity, sensitivity, and practicality of research by involving the local knowledge of the participants; (e) extending the likelihood of overcoming the distrust of research by communities that traditionally have been the 'subjects' of such research; and (f) aiming to improve health and well-being of the involved communities" [6]. CBPR provides a fundamental platform for translating science into practice and for policy development, implementation, and evaluation [2].

In practice, CBPR has been widely adopted by studies to address the urgency of translating research findings into practice, and reducing health disparities and improving health equity [2, 3, 7-9]. It addresses the challenges of translational research in the ways of (a) increasing the study's generalizability through engaging community 
stakeholders and interpreting specific findings within a cultural, organizational, and environmental context; (b) reducing the inaccuracy of one-way translation of knowledge by integrating indigenous knowledge, beliefs, and theories that can motivate the community to take a collective action for change; (c) overcoming the language barrier between the academia and the community by being linguistically and culturally sensitive; (d) preventing researchers from imposing their unidirectional research agenda on the community through sharing the power of identifying the community needs, creating research goals and objectives, making decisions, and disseminating information with the local community; (e) improving the sustainability of study impact by strengthening the sense of community members' ownership of the study and facilitating the community capacity building; and (f) alleviating the tension between researchers and underserved communities through building trustworthy, mutually benefited, and long-term partnerships [3]. With that being said, CBPR has a strong potential to reach out to more marginalized and/or racial/ethnic minority communities, respond to their health concerns, and deliver more tailored healthcare to them [3, 7].

CBPR is also a powerful tool in health policymaking to promote both procedural and distributive social justice [10-13]. As O’Brien and his colleagues identified, four mechanisms make CBPR especially crucial in the policymaking process. For instance, CBPR directly involves individuals, organizations, and communities in research, programs, and interventions as well as dissemination of results, which greatly increases the community's awareness of their health issues and the likelihood of advocating for themselves [13]. It gives an opportunity to academic researchers to obtain abundant local data and recognize key local health problems. These data can be more representative in developing policies or making policy recommendations that are specifically tailored to local concerns than using general regional or national data [13]. It provides the opportunity for community partners to help academic researchers interpret their research findings within a local context, which further enhances the researchers' ability to advocate for a policy change for their local partners [13]. Finally, CBPR builds a natural long-term infrastructure (e.g., community resources, partnerships), which may make local policymakers more likely to address a local health issue because of the motivated community and its bonding relationship with academic counterparts [13]. In Cacari-Stone's two examples, toxic free neighborhood campaign in Old Town National City, San Diego and Trade, Health, and Environment Impact Project in Los Angeles and Long Beach Ports, research findings generated in the CBPR process, were widely used in public testimonies, town hall meetings, community protests, and media advocacy, which facilitated a policy movement and changed health status of low-income communities of color who were predominantly affected by the environmental pollutants [12]. Israel and her colleagues also aimed to empower the community by facilitating community-capacity building for policy advocacy through CBPR. The Neighborhoods Working in Partnership project provided the community with a train-the-trainers program to strengthen community leaders' knowledge and skills for policy advocacy as well as competencies to train others in this arena; four workshops to train neighborhood residents in policy advocacy; and technical assistance to workshop participants in their future policy change endeavor [11]. The evaluation results of these activities were overwhelmingly positive [11]. The proposed study is to systematically examine how CBPR has been applied to improving underserved refugee populations' health and identify the successes and challenges of CBPR utilization among refugees.

\section{Materials and Methods}

\subsection{Inclusion and Exclusion Criteria}

Regardless of the research methods (e.g., qualitative, quantitative, mixed methods) and the location of the study, peer-reviewed journal articles, reports, commentaries, theses, dissertations, books, and book chapters in English that are related to CBPR and refugees' health and published until 2018 were included. Publications discussed any specific types of CBPR approaches, such as photovoice, were also included for a careful review. Refugees' nationality and geographic location were not specified in the search. Publications relevant to community-based action research, participatory action research, community-based collaborative action research, community-based intervention research, participatory research, community-participatory partnered research, community-based assessment were excluded from this review.

\subsection{Search Strategy}

The primary search included a total of 930 peer-reviewed journal articles, reports, commentaries, theses, dissertations, books, and book chapters in English, retrieved from several major databases (e.g., EBSCO, ERIC, MEDLINE Complete, MEDLINE, Medline Plus, PubMed, PsycINFO, CINAHL Plus, and Google Scholar). "Community-based participatory research and refugees" and "community-based participatory research and photovoice and refugees" were the key words used for the search. A total of 14 peer-reviewed journal articles were selected for the final data analysis. These articles contain in-depth information concerning the utilization of CBPR in addressing refugees' health disparities and its strengths and challenges. 


\subsection{Data Extraction and Synthesis}

Information was extracted from the 14 peer-reviewed journal articles and synthesized using the theme analysis. Codes were generated from the original quotes in these 14 articles and then categorized into subcategories (e.g., advocacy, shared learning, sociocultural determinants, methodological rigor, trust, stigmatization, recruitment), which all eventually fall under either strengths or challenges of utilization CBPR among refugee populations. Information relevant to the country of the research study/ program/ intervention, ethnicity of the study refugee population, and purpose of the study/ program/ intervention were also collected.

\section{Results}

All 14 peer-reviewed journal articles were published between 2007 and 2016. Eleven of them had their studies conducted in the U.S. and three were in a foreign country, including Australia, Canada, and South Korea. Five out of 14 articles targeted both refugees and immigrants. The utilization of CBPR varies from planning and implementing free tuberculosis screening, developing and evaluating a socioculturally appropriate physical activity and nutrition intervention, verifying refugees' mental and other healthcare needs, understanding refugees' healthcare-seeking behaviors, and their vulnerabilities and community cultural health assets, and describing the evolution of collaborative partnership building between academia and refugee communities [14-27].

These 14 articles described the successes and challenges of applying CBPR to addressing health issues among resettled refugee populations. What was discussed most by the current literature is that CBPR successfully enhanced the shared learning between academia and local refugee communities through frequent and open communications, including organizing community meetings, participating in networking events, and attending community cultural activities, and building the trust among them [14, 15, 21-25, 27]. Academic partners were able to better understand refugees' social and cultural perspectives on their health, their short-term and long-term health concerns, and their needs of healthcare services [14, 21-24]. As described in one study, the CBPR was "instrumental in achieving a more holistic understanding of the adolescent and young adult refugees" [24]. Local refugee communities were able to raise their awareness of their own health problems and their community health resources, learned the knowledge of research goals, objectives, and methodology, and the process of obtaining informed consent, and reduced their fear towards research [14, 21-24]. Refugee community leaders were able to "present their feedback on the project's overall objectives, recruitment strategies, consent forms, interview measures, and interview procedures" [14]. The shared understanding enabled people, such as academic researchers and service providers, to address multifaceted sociocultural determinants in refugees' health in their research $[18,20,26]$, as well as promote the methodological rigor, i.e. it increases the likelihood of researchers developing and delivering more linguistically and culturally appropriate tailored programs to address refugees' health disparities and more accurately interpret their research findings [14, 21, 25]. The time and energy invested in enhancing mutual understanding, building partnerships, and increasing community involvement in all aspect of the partnership effectively built the respect and trust among all stakeholders [14, 15, 22, 25]. Trust-building was proven to be particularly beneficial to the communities that are "often stigmatized, vulnerable, distrustful, and reluctant to participate in formalized studies using traditional research methods" [15]. One study showed the effectiveness of "addressing concerns of stigmatization raised by mandatory tuberculosis testing of foreign-born individuals" [17]. Johnson also found in their study that "the trust engendered through referrals made within the Somali community network of neighbors, acquaintances, family members, and peers enabled our team to gain entry into a close-knit social network that might otherwise have been impenetrable" [15]. Trust and community engagement made the participant recruitment more feasible [15, 17, 22, 25]. "The mobilization of support and the strength of community social networks were invaluable to the success of the community partnership. Recruitment relied exclusively on word-of-mouth, face-to-face communication" [15]. Community partners were also crucial for developing effective recruitment and retention strategies [25]. Further, the extensive community engagement promoted ethical practices [14, 25]. A review of ethical research on refugee communities and the use of CBPR indicated that the involvement of the Community Advisory Board (CAB) in the research "contributed to minimizing risks of the study, as well as ensuring that the overall assessment of benefits outweighed the risks" [14]. It increased the accuracy of data interpretation, as well as the accessibility of the data [14]. It decreased the chances of cultural misunderstanding by having a community member serving as a cultural broker [14]. CBPR also broadened the dissemination of study results and sustained the project activities $[15,16$, 18]. In a study of building partnerships with a Somali refugee community, the "preliminary findings" of the gathered data were shared and discussed with members of the research team as well as with the Somali community. As a result, Refugee and Immigrant Women's Health Initiative has remained an active initiative through Columbus Immigration Resource Center, and offers health referral services for Somali women and their families, facilitates access to healthcare services, including prenatal and reproductive health care in partnership with local healthcare providers, and distributes health information materials" [15]. Through CBPR and information exchange, 
local refugee communities were empowered to advocate for their own needs $[15,22,23]$. Communities used "research findings from the study to advocate for the allocation of additional fiscal resources and support" [22]. On the other hand, the CBPR process greatly enhanced faculty and staff, students, and practitioners' cultural competency and recognized the importance of diversity and cultural inclusion [18, 21].

However, challenges also presented during the CBPR practice [14, 15, 19, 22]. Non-probability sampling is one of them. For instance, refugees are often hard-to-reach because of cultural and linguistic differences and their previous traumatic experiences. CBPR increases the likelihood of recruiting participants through word-of-mouth and face-to-face communication [15]. Snowball sampling was often applied to surveys, interviews, and focus group. One study indicated that "as with many CBPR and qualitative methodologies, our sample was hand-picked, i.e., the Hmong Mutual Assistance Association and Planning Committee identified men and women for the focus groups and interviews. Their selection was undoubtedly subjective and they could have unknowingly pressured people to attend" [19]. CBPR in general also requires constant self-reflection on researchers themselves [22]. "All members of the research team must be learners; they must be willing to make and learn from mistakes, admit to lack of understanding and re-evaluate their approach" [22]. It can become time-consuming with constantly reviewing and revising projects based on the feedback collected from the community members and lessons learned through the process, which is a so-called repetition burden [22]. CBPR might not fit into the culture or structure of some institutions who heavily relied on traditional western clinical research "which undervalued community members' knowledge and ability to significantly contribute to the research process" [14]. "Publication and dissemination of results from studies using community participatory methods might take longer than traditional clinical research, and a lack of familiarity with community participatory methods among faculty on promotions and tenure committees might hinder junior investigators" [14]. Finally, some clinical procedures can also be perceived as being challenging. For instance, clinic exams for some sensitive health issues might not be feasible with a full-community involvement because of individuals' culture and health beliefs [15].

\section{Discussion}

Although there is a lack of explicit discussions on successes and challenges of the utilization of CBPR among refugee populations in the current literature, the findings from the refugee populations are consistent with findings from other populations. Some key successes, including the benefits of shared learning, trust building, participants' recruitment and retention, empowerment of both community and academic partners, cultural competency, results dissemination, program sustainability, and community advocacy are overwhelmingly recognized by both refugee studies conducted in different countries and studies among other populations. These end results are in compliance with the CBPR principles [14-30].

However, as CBPR is being continuously practiced, challenges remained within the refugee group along with many others [14, 15, 19, 22, 28-39]. Ethical issues are one of them [28, 31-33]. On one hand, the current findings demonstrated the success of the involvement of $\mathrm{CAB}$ in assuring to minimize study risks and maximize its benefits at both individual and community levels. $\mathrm{CAB}$ strengthened both the accuracy of data interpretation and the accessibility of study results to the community [14]. Human Subjects Protection Training for community members increased the individuals' awareness of ethics of conducting research and assured ethical recruitment of a study [25]. On the other hand, because of the nature of CBPR, researchers questioned if the currently used individual level consent form was sufficient to assure the community's interests, if community members had the right to ask why their community was selected and if the community as a whole could decide whether they would like to participate and who should make that decision on behalf of community's interests [33]. Researchers also questioned if a study could truly serve the mutual benefits of both the community and the academia as CBPR advocated while still maintained the rigor of study methodology [28, 31, 33]. Lantz et al. identified the challenge of achieving a balance between community interests and research needs [31]. Community members could be more interested in a community health intervention which could lead to a systematic change of their community rather than just being the study subjects while academic researchers might be more interested in "gaining generalizable knowledge and understanding through etiologic and evaluation research" [31]. It was also difficult in achieving the agreement on things, such as selection of research questions, study design, and data collection measurements because of the lack of the depth of the shared understanding [28]. Community health interventions using randomized control trails became even more limited in CBPR researches due to ethical, structural, and political considerations [28, 33]. Through Bastida et al. practice of CBPR, six general principles were suggested to address the ethical concerns, including (1) develop respect among all partners throughout the course of research; (2) maintain the transparency of every effort made to the study; (3) build a strong sense of fairness among all stakeholders which is critical to building trust; (4) assure the community's full understanding of the informed consent and their being voluntary in the participation of the study; (5) maintain the reciprocity of interactions among all study partners as study goals, methods, and responsibilities are 
being discussed; and (6) facilitate equal voice of all partners and a full disclosure of study results [32].

The institution-related cultural and structural barriers to CBPR practice identified by the current study with refugee populations concurred with the previous findings among non-refugee study populations [14, 34-36]. In addition to the examples indicated by the current study, other researchers also emphasized the institutional inflexibility of giving equitable recognition of co-investigators and appropriate fiscal arrangement [35]. CBPR promotes equity among all stakeholders, which leads to the question of who should be allowed to be the principal or co-investigators, community members, academic researchers, or both? Institutional policies could prohibit the non-traditional arrangement of principal or co-investigators [35]. It also takes a long time to build robust community partnerships to implement CBPR. It could take even a longer time to publish. Because of the collaborative nature of CBPR, co-authorships in publications can be common in CBPR researches. Institutions that did not support faculty practice time in CBPR and placed a high value on single authorship in publications could be very detrimental to both CBPR practice and faculty tenure and promotion [34, 36]. Structural and cultural changes have been suggested at the institutional level. Some universities were moving toward changing criteria for tenure and promotion to support their faculty and students to be more involved in CBPR research $[34,36]$. It could also be beneficial to improve the administration's awareness of CBPR to foster a campus-wide culture of community engagement in research [35]. Moreover, partnership building could also become very challenging [22. 37-39]. For example, both academic partners and community partners could hold stereotypes towards each other. Academic partners might not believe that community partners were well equipped for achieving the research aims of their project, and community partners might believe that academic partners conducting research and being interested in collaboration was simply because they wanted to advance their career, publish their papers, and get promoted [38]. The beliefs on these stereotypes could create tension and power imbalance in CBPR and lead to distrust [38]. The time and resources, especially financial resources, invested in both building and sustaining the partnerships could also be tremendous [22, 34, 39]. As indicated by the current study, it was so common for researchers to constantly spend time reflecting on themselves and their project and repeatedly reviewing and revising their project throughout all phases of CBPR, which required significant amount of time and commitment, which might not even be supported by the project funding and required subsequent funding applications [22]. It makes even more crucial to have both institutional and sustained funding support, and maintain an active and clear communication and have more committed people at the table [39].

\section{Conclusions}

Both successes and challenges of utilizing CBPR identified in the current study were not unique to refugee study populations. They were shared by many other non-refugee study populations. Successes were made in areas, such as shared learning, trust, participants' recruitment, methodological rigor, results dissemination, project sustainability, advocacy, and cultural competency [14-30]. Challenges also remained to improve the areas, including ethics, institutional structures, partnership building, and time and funding support [14, 15, 19, 22, 31-39], which require institutional cultural, structural, and policy changes as well as the recognition of the nature of CBPR in both external and internal funding mechanisms [32, 34, 36, 39].

\section{REFERENCES}

[1] M. Viswanathan A. Ammerman, E. Eng, G. Gartlehner, K. N. Lohr, D. Griffith, S. Rhodes, C. Samuel-Hodge, S. Maty, L. Lux, L. Webb. Community-based participatory research: Assessing the evidence. Evidence Report/Technology Assessment, No, 99, 1-8.

[2] E. L. Baker, L. E. White, M. Y. Lichtveld. Reducing health disparities through community-based research. Public Health Reports, Vol. 116, No. 6, 517-519.

[3] N. Wallerstein, Duran B. Community-based participatory research contributions to intervention research: The intersection of science and practice to improve health equity. American Journal of Public Health. Vol. 100, No. S1, S40-S46.

[4] Z. Faridi, J. A. Grunbaum, B. S. Gray, A. Franks, E. Simoes. Community-based participatory research: necessary next steps. Preventing Chronic Disease. Vol. 4, No. 3, A70-A74.

[5] D. L. Katz. Representing your community in community-based participatory research: Differences made and measured. Preventing Chronic Disease. Vol. 1, No. 1, A12-A15.

[6] B. A. Israel, E. A. Parker, Z. Rowe, A. Salvatore, M. Minkler, J. López, A. Butz, A. Mosley, L. Coates, G. Lambert, P. A. Potito. Community-based participatory research: Lessons learned from the Centers for Children's Environmental Health and Disease Prevention Research. Environmental Health Perspectives. Vol. 113, No, 10, 1463-1471.

[7] D. De Las Nueces, K. Hacker, A. DiGirolamo, L. S. Hicks. A systematic review of community - based participatory research to enhance clinical trials in racial and ethnic minority groups. Health Services Research. Vol. 47, No. 3pt2, 1363-1386.

[8] M. U. Shalowitz, A. Isacco, N. Barquin, E. Clark-Kauffman, P. Delger, D. Nelson, A. Quinn A, K. A. Wagenaar. Community-based participatory research: A review of the literature with strategies for community engagement. 
Journal of Developmental \& Behavioral Pediatrics. Vol. 30, No. 4, 350-361.

[9] W. K. Cook. Integrating research and action: A systematic review of community-based participatory research to address health disparities in environmental and occupational health in the USA. Journal of Epidemiology \& Community Health. Vol. 62, No. 8, 668-676.

[10] D. Petersen, M. Minkler, V. B. Vásquez, A. C. Baden. Community - Based Participatory Research as a Tool for Policy Change: A case study of the southern California environmental justice collaborative. Review of Policy Research. Vol. 23, No. 2, 339-354.

[11] B. A. Israel, C. M. Coombe, R. R. Cheezum, A. J. Schulz, R. J. McGranaghan, R. Lichtenstein, A. G. Reyes, J. Clement, A. Burris. Community-based participatory research: A capacity-building approach for policy advocacy aimed at eliminating health disparities. American Journal of Public Health. Vol. 100, No. 11, 2094-2102.

[12] L. Cacari-Stone, N. Wallerstein, A. P. Garcia, M. Minkler. The promise of community-based participatory research for health equity: A conceptual model for bridging evidence with policy. American Journal of Public Health. Vol. 104, No. 9, 1615-1623.

[13] M. J. O’Brien, R. C. Whitaker. The role of community-based participatory research to inform local health policy: A case study. Journal of General Internal Medicine. Vol. 26, No. 12, 1498-1501.

[14] B. H. Ellis, M. Kia-Keating, S. A. Yusuf, A. Lincoln, A. Nur. Ethical research in refugee communities and the use of community participatory methods. Transcultural Psychiatry. Vol. 44, No. 3, 459-481.

[15] C. E. Johnson, S. A. Ali, M. P. L. Shipp. Building community-based participatory research partnerships with a Somali refugee community. American Journal of Preventive Medicine. Vol. 37, No. 6, S230-S236.

[16] P. J. Springer, M. Black, K. Martz, C. Deckys, T. Soelberg. Somali Bantu refugees in southwest Idaho: Assessment using participatory research. Advances in Nursing Science. Vol. 33, No. 2, 170-181.

[17] M. L. Wieland, J. A. Weis, M. W. Olney, M. Alemán, S. Sullivan, K. Millington, C. O'hara, J. A. Nigon, I. G. Sia. Screening for tuberculosis at an adult education center: Results of a community-based participatory process. American Journal of Public Health. Vol. 101, No. 7 , 1264-1267.

[18] D. Bates, E. Burman, L. Ejike-King, C. Rufyiri. Healthy transitions: A community-based participatory research approach with Burundians with refugee status. Journal of Higher Education Outreach and Engagement. Vol. 16, No. 3, 153-174.

[19] A. F. Collier, M. Munger, Y. K. Moua. Hmong mental health needs assessment: A community-based partnership in a small mid-western community. American Journal of Community Psychology. Vol. 49, No. 1-2, 73-86.

[20] M. L. Wieland, J. A. Weis, T. Palmer, M. Goodson, S. Loth, F. Omer, A. Abbenyi, K. Krucker, K. Edens, I, G. Sia. Physical activity and nutrition among immigrant and refugee women: A community-based participatory research approach. Women's Health Issues. Vol. 22, No. 2, e225-e232.

[21] C. C. Sonn, M. Grossman, A. Utomo. Reflections on a participatory research project: Young people of refugee background in an arts-based program. Journal for Social Action in Counseling \& Psychology. Vol. 5, No. 3, 95-110.

[22] R. M. Campbell, A. G. Klei, B. D. Hodges, D. Fisman, S. A. Kitto. Comparison of health access between permanent residents, undocumented immigrants and refugee claimants in Toronto, Canada. Journal of Immigrant and Minority Health. Vol.16, No. 1, 165-176.

[23] T. S. Betancourt, R. Frounfelker, T. Mishra, A. Hussein, R. Falzarano. Addressing health disparities in the mental health of refugee children and adolescents through community-based participatory research: a study in 2 communities. American Journal of Public Health. Vol. 105, No. S3, S475-S482.

[24] M. A. Kim, J. S. Hong, M. Ra, K. Kim. Understanding social exclusion and psychosocial adjustment of North Korean adolescents and young adult refugees in South Korea through Photovoice. Qualitative Social Work. Vol. 14, No. 6, 820-841.

[25] M. M. Hanza, M. Goodson, A. Osman, M. D. P. Capetillo, A. Hared, J. A. Nigon, S. J. Meiers, J. A. Weis, M. L. Wieland, I. G. Sia. Lessons learned from community-led recruitment of immigrants and refugee participants for a randomized, community-based participatory research study. Journal of Immigrant and Minority Health. Vol.18, No. 5, 1241-1245.

[26] E. Lightfoot, J. Blevins, T. Lum, A. Dube. Cultural health assets of Somali and Oromo refugees and immigrants in Minnesota: Findings from a community-based participatory research project. Journal of Health Care for the Poor and Underserved. Vol. 27, No. 1, 252-260.

[27] B. C. Reimers. Peacebuilding in refugee resettlement communities: Using photovoice to find common ground. Journal of Peacebuilding \& Development. Vol. 11, No. 3, 108-113.

[28] E. Kobetz, J. Menard, J. Diem, B. Barton, J. Blanco, L. Pierre, P. D. Auguste, M. Etienne, C. Brewster, C. Community-based participatory research in Little Haiti: Challenges and lessons learned. Progress in Community Health Partnerships: Research, Education, and Action. Vol. 3, No. 2, 133-137.

[29] Y. Salimi, K. Shahandeh, H. Malekafzali, N. Loori, A. Kheiltash, E. Jamshidi, A. S. Frouzan, R. Majdzadeh. Is community-based participatory research (CBPR) useful? A systematic review on papers in a decade. International journal of preventive medicine. Vol. 3, No. 6, 386-392.

[30] M. Minkler. Community-based research partnerships: Challenges and opportunities. Journal of Urban Health. Vol. 82, No. 2, ii3-ii12.

[31] P. M. Lantz, E. Viruell-Fuentes, B. A. Israel, D. Softley, R. Guzman. Can communities and academia work together on public health research? Evaluation results from a community-based participatory research partnership in Detroit. Journal of Urban Health. Vol. 78, No. 3, 495-507.

[32] E. M. Bastida, T. S. Tseng, C. McKeever, L. Jack Jr, Ethics 
and community-based participatory research: Perspectives from the field. Health Promotion Practice. Vol. 11, No. 1, 16-20.

[33] D. R. Buchanan, F. G. Miller, N. Wallerstein. Ethical issues in community-based participatory research: balancing rigorous research with community participation in community intervention studies. Progress in community health partnerships: Research, education, and action. Vol. 1, No. 2, 153-160.

[34] C. J. Strickland. Challenges in community-based participatory research implementation: Experiences in cancer prevention with Pacific Northwest American Indian tribes. Cancer Control. Vol. 13, No. 3, 230-236.

[35] J. Shoultz, M. F. Oneha, L. Magnussen, M. M. Hla, Z. Brees-Saunders, M. D. Cruz, M. Douglas. Finding solutions to challenges faced in community-based participatory research between academic and community organizations. Journal of Interprofessional Care. Vol. 20, No. 2, 133-144.

[36] N. B. Wallerstein, B. Duran. Using community-based participatory research to address health disparities. Health Promotion Practice. Vol. 7, No. 3. 312-323.

[37] L. Burhansstipano, S. Christopher, A. Schumacher Sr, Lessons learned from community-based participatory research in Indian country. Cancer Control. Vol. 12, No. 4_suppl, 70-76.

[38] E. R. Freeman, D. Brugge, W. M. Bennett-Bradley, J. I. Levy, E. R. Carrasco. Challenges of conducting community-based participatory research in Boston's neighborhoods to reduce disparities in asthma. Journal of Urban Health. Vol. 83, No. 6, 1013-1021.

[39] B. A. Israel, J. Krieger, D. Vlahov, S. Ciske, M. Foley, P. Fortin, J. R. Guzman, R. Lichtenstein, R. McGranaghan, A. G. Palermo, G. Tang. Challenges and facilitating factors in sustaining community-based participatory research partnerships: Lessons learned from the Detroit, New York City and Seattle Urban Research Centers. Journal of Urban Health. Vol. 83, No. 6, 1022-1040. 